\title{
Regulação da Saúde Suplementar e Estrutura Etária dos Beneficiários
}

\author{
Supplementary Health Care Regulation \\ and Age Structure of Beneficiaries
}

Matheus Stivali ${ }^{1}$

\begin{abstract}
The paper exposes the changes in rules of price readjustment of health plans by age thresholds and demographic changes observed between 1998 and 2008. By calculating aging indicators and building population pyramids, it assesses whether the demographic changes coupled with the regulation caused any alteration in young people subscribing to supplementary healthcare plans. The indicators reveal the aging trend of beneficiaries of health plans, especially among those contracted individually, and also that this has not resulted in young people quitting supplementary healthcare plans.

Key Words Supplementary health, Prepaid health plans, Demographic aging, Government regulation
\end{abstract}

Resumo $O$ artigo expõe as mudanças das regras de reajuste das contraprestações por faixas etárias assim como as mudanças demográficas observadas entre 1998 e 2008. Avaliam-se, através do cálculo de indicadores de envelhecimento e da construção de pirâmides etárias, se as mudanças demográficas e a regulação provocaram alguma alteração na adesão da população jovem à saúde suplementar. Os indicadores mostram o envelhecimento populacional entre os beneficiários de planos de saúde, em especial entre os com planos de contratação individual, e também que isso não implicou abandono da saúde suplementar pelos jovens.

Palavras Chave Saúde suplementar, Planos de pré-pagamento em saúde, Envelhecimento da população, Regulamentação governamental
${ }^{1}$ Instituto de Pesquisa Econômica Aplicada - IPEA. SBS Quadra 1 - Bloco J -

Edifício BNDES - Sala 1605. 70076-900 Brasília DF. matheus.stivali@ipea.gov.br 


\section{Introdução}

A intensificação da mudança na estrutura etária da população brasileira apresenta uma série de desafios para a saúde suplementar, assim como para os outros sistemas de proteção social do País. As edições de 1998 e de 2003 do suplemento saúde da Pesquisa Nacional por Amostra de Domicílios, do Instituto Brasileiro de Geografia e Estatística, (Pnad-IBGE) coincidem com mudanças importantes na regulação dos planos de saúde, a saber, a Lei ${ }^{\circ} 9.656 / 98^{1}$ e a Lei ${ }^{\circ} 10.741 / 03^{2}$ (Estatuto do Idoso).

Por meio dessas Leis foi introduzida na regulação do setor, entre outras normas, a variação das contraprestações segundo as faixas etárias dos beneficiários. Essa variação ocorre respeitando certas limitações, que correspondem à solidariedade intergeracional do sistema de saúde suplementar, isto é, um subsídio obrigatório das faixas etárias mais jovens para as mais velhas. $\mathrm{O}$ objetivo dessa regulação é limitar a capacidade das operadoras de discriminar, por idade, o valor das contraprestações pagas pelos beneficiários de planos de saúde, impondo um elemento de equidade ao sistema.

Essa regra é relevante para o funcionamento da saúde suplementar, pois, do ponto de vista econômico, os planos de saúde são uma modalidade de seguro, isto é, em caso de adoecimento, a operadora de planos garante assistência à saúde ao segurado (beneficiário), tendo como contrapartida o pagamento contínuo de prêmios (contraprestações). Para que o financiamento saúde suplementar funcione adequadamente é necessário que as operadoras de planos de saúde mantenham seu equilíbrio atuarial, cada operadora deve ter um conjunto de beneficiários - chamado de pool de risco - equilibrado. Se o conjunto de beneficiários for composto apenas por pessoas que usam intensivamente a assistência à saúde, o plano será incapaz de arcar com as despesas.

O envelhecimento populacional implica justamente na alteração da composição do pool de risco das operadoras e, segundo a previsão teórica dos modelos microeconômicos ${ }^{3,4}$, isso criaria tensões nas regras de solidariedade intergeracional existentes na regulação do setor.

Completados dez anos da Lei n ${ }^{\circ} 9.656 / 98^{1} \mathrm{em}$ 2008 uma série de avaliações da regulação da saúde suplementar já foi realizada, destacadamente Cechin $^{5}$, Santos et al. ${ }^{6}$ e Montone ${ }^{7}$. O objetivo deste trabalho é avaliar a validade da previsão teórica supramencionada, aprofundando a análise dos efeitos da regulação da saúde suplemen- tar, especificamente da regra de reajuste segundo faixa etária do beneficiário. Para tanto se realiza analise sócio-demográfica dos beneficiários a partir das informações coletadas pelas edições de 1998, 2003 e 2008 do suplemento saúde da Pnad-IBGE.

\section{Seguros, Planos de Saúde e o Compartilhamento de Risco}

No funcionamento de um seguro tradicional como um seguro de automóveis, por exemplo não há compartilhamento de riscos, o que implica que os indivíduos com maior risco de sinistro - no exemplo, maior risco de ter o carro roubado ou danificado - paguem prêmios maiores. No caso dos cuidados à saúde, os riscos estão associados a características inerentes dos beneficiários e refletem sua possibilidade de adoecer ou, do ponto de vista da operadora, gerar despesas (ademais, do lado da oferta, pode-se citar a rede de prestadores vinculada à operadora como um determinante importante do valor das contraprestações).

Como o acesso a bens e serviços de saúde é considerado um bem meritório, os seguros de saúde não funcionam totalmente como um seguro tradicional e envolvem sempre algum grau de compartilhamento de riscos, sendo um determinante importante do valor das contraprestações a forma como essa divisão é feita.

As principais formas de incorporar o risco na determinação das contraprestações de planos de saúde são: : (1) Community rating: Sistema de fixação dos prêmios que exige que todos os segurados (de uma mesma região, de uma mesma empresa, de um mesmo plano) paguem valores iguais pelo mesmo plano. $\mathrm{O}$ valor do prêmio depende então do risco médio do grupo (pool de risco), há compartilhamento total de risco. (2) Modified Community rating: É como o community rating puro, mas é permitida alguma variação nos prêmios segundo um critério pré-estabelecido (idade e/ou sexo, por exemplo). O valor do prêmio depende então do risco médio de cada subgrupo e de quanto um subgrupo subsidia o outro, há compartilhamento parcial de risco. (3) Experience rating: Sistema de fixação dos prêmios que se baseia no uso do seguro pelo consumidor (isto é, quanto mais o plano for utilizado, maior será seu custo nos períodos seguintes). $\mathrm{O}$ valor do prêmio depende das características risco e do histórico médico do segurado, não há compartilhamento de risco. 
A formação do pool de risco garante que o pagamento das consultas e cirurgias efetivamente realizadas seja rateado entre todos vinculados ao plano, independentemente de seu estado de saúde, e não apenas pelo beneficiário que adoecer. Já o compartilhamento de risco tem o objetivo de garantir que pessoas com más condições de saúde - que teriam que pagar prêmios muito elevados em um esquema tradicional de seguro - também consigam acessar a bens e serviços para o seu tratamento.

Nos mercados de seguro, em geral, há a presença de risco moral. Ou seja, por conta dos incentivos gerados pelo seguro, é introduzido o risco de que o segurado passe a se comportar de maneira diferente do que na ausência do seguro. No setor de cuidados à saúde, esse fato é agravado pela presença de assimetria de informação entre os diversos agentes envolvidos: consumidores (beneficiários), operadoras, médicos e provedores (hospitais, laboratórios etc...).

A combinação da informação assimétrica com situações em que as operadoras são obrigadas a cobrar um mesmo valor de contraprestação gera a chamada seleção adversa, que corresponde aos planos mais generosos (maior cobertura de procedimentos ou maior rede de prestadores, por exemplo) terem desproporcionalmente mais segurados com pior estado de saúde enquanto os planos menos generosos tem desproporcionalmente mais segurados com melhor estado de saú$\mathrm{de}^{1}$. Isso incentiva as seguradoras a tentarem selecionar seus clientes de forma a compor o pool mais saudável possível, realizando a chamada de seleção de risco. É esta característica deste mercado que justifica a regulação proibindo a discriminação dos beneficiários. Neste contexto, a seleção adversa e a seleção de risco são inerentes ao setor de seguro saúde que sempre envolve, em algum grau, o compartilhamento de riscos.

No modelo de referência para a análise microeconômica de seguro-saúde ${ }^{3,4}$, que condensa os conceitos descritos acima, há permanentemente um trade-off entre a eficiência do mercado e a equidade, determinado pela capacidade de discriminação dos beneficiários segundo seus riscos pelas operadoras. Sendo um dos resultados possíveis o colapso do mercado (devido a uma "espiral de seleção adversa”) no caso da imposição de um mesmo valor de contraprestações (mensalidade) para todos os beneficiários de planos?.

No caso da saúde suplementar no Brasil, a regulação impede a discriminação entre os segurados por parte das operadoras, sendo possível cobrar prêmios diferentes por um mesmo pro- duto apenas entre grupos etários diferentes. Essa regulação tem como marcos a Lei no 9.656, de 3 de junho de 1998, e a criação da Agência Nacional de Saúde Suplementar (ANS), Lei no 9.961루, de 28 de janeiro de 2000. Nos anos seguintes essas leis foram alteradas por uma série de medidas provisórias, alcançando relativa estabilidade após o ano 2000.

Quanto às variações do valor das contraprestações, a regulação do setor estabelece que elas possam ser ocasionadas por três diferentes motivos: variação por faixa etária, reajuste por variação de custo e reequilíbrio econômico financeiro do contrato. Devido ao primeiro motivo, o financiamento na saúde suplementar brasileira é chamado de mutualismo com solidariedade intergeracional (que corresponde ao modified community rating). Por mutualismo entende-se que, dentro de cada faixa etária, o prêmio é igual para todos os membros daquele plano e é determinado pelo risco médio daquele grupo. Ou seja, os indivíduos mais saudáveis (de menor risco) subsidiam aqueles menos saudáveis (de maior risco), dentro da mesma faixa etária. Por solidariedade intergeracional, entende-se que os segurados das faixas etárias mais jovens (de menor risco) subsidiam os indivíduos mais idosos (de maior risco). Além disso, na regulação da saúde suplementar há uma limitação no quanto os prêmios podem ser diferentes entre as faixas etárias de um mesmo plano.

A Lei n⿳0 9.656/98 e a Resolução no 6, de 4 de novembro de $1998^{1}$, do Conselho de Saúde Suplementar (Consu), já estabeleciam faixas etárias e uma diferença máxima permitida entre o prêmio cobrado da primeira e o da última faixa etária. A divisão original era feita em sete grupos etários, com tamanho de dez anos, essa divisão possibilitava uma maior heterogeneidade entre os beneficiários que pertenciam a uma mesma faixa etária. Ademais, o estabelecimento do limite máximo de diferença entre os prêmios da última e da primeira faixa implica o estabelecimento de um subsídio mínimo das faixas mais jovens para as mais idosas.

Destaque-se que, desde essa primeira versão, as regras determinam não só a existência das faixas etárias, mas também que não podem ser aplicados aumentos a pessoas com mais de sessenta anos que tenham permanecido no plano por mais de dez anos. Este tipo de dispositivo age como um incentivo aos consumidores participarem do esquema de subsídio entre diferentes grupos etários, garantindo um pagamento menor no futuro em troca de um pagamento maior no presente. 
A Lei $n^{\circ} 10.741$, de $1^{\circ}$ de outubro de $2003^{2}$ chamada de Estatuto do Idoso, vedou a variação de preços dos planos em razão da idade para todos os maiores de sessenta anos. Com isso, a regulação do setor precisou se adaptar, o que foi feito pela Resolução Normativa da ANS no 63, de 20 de dezembro de 2003. Nessa nova regra foram estabelecidas novas faixas e limites adicionais para a variação dos prêmios: entre a primeira e a sétima faixa e entre a sétima e a décima faixa. As duas regras para faixas etárias são resumidas abaixo.

Como pode ser observado na Tabela 1, a nova divisão de faixas etárias correspondeu à divisão de grupos mais homogêneos (i.e., com menor diferencial de idade) e ao rebaixamento da faixa etária mais alta provocada pelo Estatuto do Idoso. Novamente isso equivale ao estabelecimento de um subsídio mínimo entre as faixas etárias, mas essa definição é mais complexa que a original. Há subsídio mínimo entre: a primeira e a sétima faixa, a primeira e a última faixa, e também entre a sétima e a última faixa.

Essas mudanças tendem ao aumento dos prêmios pagos pelas faixas etárias mais jovens frente ao valor que seria pago nas regras anteriores.

Tabela 1. Diferencial máximo permitido entre as contraprestações (prêmios) de planos de saúde para contratos assinados

\begin{tabular}{|c|c|c|c|}
\hline \multicolumn{2}{|c|}{$\begin{array}{c}\text { Entre } 01 / 01 / 1999 \\
\text { e } 31 / 12 / 2003\end{array}$} & \multicolumn{2}{|c|}{$\begin{array}{c}\text { A partir } \\
\text { de } 01 / 01 / 2004\end{array}$} \\
\hline $\begin{array}{l}\text { Faixas } \\
\text { etárias }\end{array}$ & Limitação & $\begin{array}{l}\text { Faixas } \\
\text { etárias }\end{array}$ & Limitações \\
\hline$[70,+]$ & $\begin{array}{l}\text { Valor máximo da } \\
\text { contraprestação } \\
=6 \mathrm{X}\end{array}$ & {$[59,+]$} & $\begin{array}{l}\text { Valor máximo da } \\
\text { contraprestação = } \\
6 \mathrm{X}=2,45 \mathrm{Y}\end{array}$ \\
\hline$[60,69]$ & & & \\
\hline$[50,59]$ & & $\begin{array}{l}{[54,58]} \\
{[49,53]}\end{array}$ & \\
\hline$[40,49]$ & & {$[44,48]$} & $\begin{array}{l}\text { Valor máximo da } \\
\text { contraprestação }= \\
\mathrm{Y}=2,45 \mathrm{X}\end{array}$ \\
\hline & & {$[39,43]$} & \\
\hline$[30,39]$ & & $\begin{array}{l}{[34,38]} \\
{[29,33]}\end{array}$ & \\
\hline$[18,29]$ & & $\begin{array}{l}{[24,28]} \\
{[19,23]}\end{array}$ & \\
\hline$[0,17]$ & $\begin{array}{c}\text { Valor } \\
\text { contraprestação } \\
=\mathrm{X}\end{array}$ & {$[0,18]$} & $\begin{array}{l}\text { Valor } \\
\text { contraprestação = } \\
\mathrm{X}\end{array}$ \\
\hline
\end{tabular}

Fonte: Elaborado a partir de ANS-Res. Consu 06/1998 e ANS-RN 63/2003.
Tudo mais constante, o envelhecimento populacional estaria justamente modificando o peso relativo dos diferentes grupos de risco, no caso, determinado pelas faixas etárias, na carteira das operadoras. A sustentabilidade do esquema de financiamento dependeria então da tolerância dos consumidores mais jovens ao nível de subsídio cruzado imposto pela regulação, e do quanto o peso desses grupos, idosos e jovens, será diferente.

Deve-se atentar que, embora as regras de faixa etárias sejam legalmente aplicáveis para todos planos de saúde regulados pela Lei no 9.656/98 ${ }^{1}$, essa é uma cláusula contratual que está presente nos planos de contratação coletiva, mas que usualmente não é aplicada, efetivando-se um preço único por beneficiário. Essa prática, documentada pelo estudo de $\operatorname{Slivak}^{10}$, aponta que para os planos de contratação coletiva as regras de reajuste de contraprestações por faixas etárias são, em termos econômicos, uma restrição não efetiva (non-binding restriction) para as decisões das operadoras. Assim, essas regras devem ser analisadas considerando separadamente os beneficiários de planos nos quais elas são sempre aplicadas (contratação individual e/ou coletivo por adesão) dos em que sua aplicação não é efetiva (contratação coletiva).

Para avaliar os efeitos da regulação se recorrerá à análise demográfica. Busca-se avaliar se há uma sobrerrepresentação de pessoas mais velhas entre os beneficiários ou se há saída de pessoas mais novas da saúde suplementar, o que representaria um efeito não intencional da regulação.

\section{Dados Utilizados e Procedimentos Adotados}

Para as analises foram utilizados os dados das três últimas edições da Pnad-IBGE que dispunham do suplemento saúde (1998, 2003 e 2008). Como a partir de 2004 a cobertura da Pnad foi expandida, considerou-se a cobertura geográfica comum às três edições (excluindo as áreas rurais da região Norte da Pnad 2008) tornando possível a comparação.

Para identificar as pessoas cobertas por planos de saúde (de assistência médico-hospitalar) foram utilizadas as variáveis V1321 e V1344, considerando "sem plano" as pessoas que possuíam planos exclusivamente odontológicos.

Quanto à forma de contratação, foi utilizada como proxy a variável V1332 referente à forma de pagamento do plano, esta informação só é disponível para o titular do plano de saúde, sendo necessária sua imputação para os dependen- 
tes. Assume-se que os beneficiários de planos coletivos têm o plano pago pelo empregador do titular, ou através do trabalho atual ou anterior do titular, com as outras possibilidades sendo consideradas como contratação individual. Essa abordagem imperfeita, contudo, é única possibilidade de investigar a forma de contratação $0^{11,12}$, já que esse ponto não é investigado diretamente pela Pnad.

Ademais, existe uma variável na Pnad (V1322) que permitiria distinguir os beneficiários de planos contratados no mercado dos destinados a servidores públicos, organizados por institutos públicos de assistência à saúde na forma de pessoa jurídica de direito público e, por isso, excluídos da regulação ${ }^{7}$. Como pode haver confusão na resposta a essa pergunta entre os planos dedicados aos servidores e planos contratados no mercado (por uma instituição representativa de servidores, contratando um plano coletivo por adesão, por exemplo) optou-se por não utilizar a informação dessa variável.

Para a análise da estrutura etária da população, esta foi dividida segundo as faixas etárias vigentes para o reajuste dos planos de saúde (detalhadas na Tabela 1). Consideraram-se ainda faixas adicionais, também com amplitude de quatro anos, para as pessoas com idade igual ou inferior a dezoito anos e para as com idade igual ou superior a cinquenta e nove anos.

Para avaliar a cobertura dos planos de saúde na população optou-se por apresentar apenas as estimativas pontuais da mesma. Essa opção foi feita devido à cobertura representar uma proporção da população geral e, devido ao tamanho de amostra da Pnad, os intervalos de confiança para essas proporções têm amplitude muito pequena, não alterando as considerações feitas sobre as estimativas pontuais.

Para os cálculos da razão de dependência (RD) e do índice de envelhecimento (IE) foram utilizados os intervalos etários usuais na demografia, e ainda dados das edições de 1978 e 1988 da Pnad para obter um horizonte temporal demograficamente relevante. $\mathrm{A} \mathrm{RD}$ corresponde à razão entre o total da população em idade não-ativa (com idade inferior a quinze ou superior a sessenta e quatro anos) e o total da população em idade ativa (com idade entre quinze e sessenta e quatro anos) $\mathrm{RD}=\frac{P_{0-14}+P_{65+}}{P_{15-64}}$.

Já o IE foi obtido pela razão entre o total da população acima de sessenta e quatro anos e o total da população com menos de quinze anos, isto é: $I E=\frac{P_{65+}}{P_{0-14}}$.
Para o cálculo das prevalências de morbidades auto-referidas foram utilizadas as variáveis relativas ao câncer (V1311), à diabetes (V1312) e à hipertensão (V1314), utilizando todas as observações com informações válidas (excluíramse as sem resposta). Como limitação do uso dessas variáveis tem-se que as informações não são diretamente comparáveis para as três edições da Pnad, devido a uma mudança nas perguntas entre 1998 e os anos seguintes. Enquanto o questionário de 1998 perguntava ao entrevistado se ele possuía determinada morbidade, nos anos de 2003 e 2008 passou-se a perguntar ao entrevistado se algum médico já havia dito que ela tinha determinada morbidade.

Adicionalmente, o valor dessas variáveis provém de informações referidas pelos entrevistados. A doença pesquisada, a presença de co-morbidades, bem como as características sociais e demográficas do respondente podem afetar a qualidade dessa informação ${ }^{13}$. Nesse trabalho não são investigados possíveis diferenciais segundo uso de outro respondente para a entrevista, o que é outra fonte potencial de erro ${ }^{14}$.

De forma complementar aos dados da Pnad foram utilizados dados extraídos do sítio da internet ANS (ANS Tabnet), sempre se considerando o mês de setembro como referência. Quanto aos dados da ANS deve-se levar em conta que a agência foi criada no ano 2000, quando já existiam vários contratos de planos em vigor, assim, é natural observar que os dados dos primeiros anos de funcionamento da agência não tenham boa qualidade (com planos com modalidade ou faixa etária não identificada) melhorando sua consistência nos anos seguintes. Deve-se atentar que os dados da ANS referem-se a vínculos com planos de saúde, podendo existir mais de um vínculo para uma mesma pessoa.

\section{Resultados - Envelhecimento Populacional e Envelhecimento das Carteiras}

O fenômeno chamado de transição demográfica corresponde à mudança nos indicadores de mortalidade e fecundidade de uma população. Estes, que inicialmente se encontram em altos níveis, passam para baixos níveis. Esta mudança não é simultânea, ocorrendo primeiro a queda da mortalidade, com consequente aumento da expectativa de vida, e, com defasagem de alguns períodos, queda pronunciada na taxa de fecundidade.

Embora os dois eventos sejam importantes, a queda na fecundidade tem maior impacto sobre a mudança da estrutura etária da popula- 
ção ${ }^{15}$. Na Tabela 2, pode-se observar a evolução de dois indicadores da estrutura etária do Brasil: a razão de dependência e o índice de envelhecimento, para o toda população e para a população coberta por planos de saúde. A transição demográfica é caracterizada pela queda do primeiro e elevação do segundo.

Por esses indicadores nota-se o avanço da transição demográfica na população brasileira, nos últimos quarenta anos a estrutura etária do Brasil se modificou de um cenário em que crianças e jovens eram a maioria da população para um cenário em que a população em idade economicamente ativa corresponde à maioria da população. Também nota-se que a parte da população que é coberta por planos de saúde é mais envelhecida que a população em geral, com uma $\mathrm{RD}$ próxima, mas com um IE bastante superior a partir de 2003.

Há ainda uma diferença significativa entre os beneficiários de planos de contratação coletiva e de contratação individual, com o último grupo correspondendo a uma população mais envelhecida e com uma trajetória crescente para a RD nos anos considerados. Esse diferencial expõe como esses dois grupos são diferentes.

A mudança na composição etária da população tem impactos diretos na estrutura de receitas e despesas da saúde suplementar, sendo um fator importante para seu planejamento de longo prazo. Do lado das receitas, tem-se a regulação do setor, que pressupõe um subsídio dos mais jovens para os mais velhos; do lado das despesas, tem-se que uma população mais idosa, em relação a uma população mais jovem, demanda não só quantidades, mas também tipos diferentes de bens e serviços de saúde.

Do ponto de vista estritamente demográfico, após a queda da fecundidade e a consequente queda na razão de dependência, chega-se ao momento com maior potencial para a expansão da cobertura da saúde suplementar, que corresponde ao que os demógrafos convencionaram chamar de bônus demográfico ${ }^{16-18}$.

Com o esgotamento do bônus demográfico a razão de dependência voltará a se elevar no futuro, mas o mesmo não acontecerá com o índice de envelhecimento, que se manterá elevado, demonstrando a predominância de idosos na sociedade.

Dado que a principal forma de acesso à saúde suplementar é por meio do vínculo empregatício (especialmente para os planos de contratação coletiva), esse seria o momento em que mais pessoas poderiam acessar a saúde suplementar, a depender de sua inclusão no mercado de trabalho. O bom desempenho do mercado de trabalho nos anos recentes elevou continuamente o número de vínculos com planos de saúde, segundo os dados da ANS, de 31.014.113 vínculos em setembro de 2000 chegou-se a $41.932 .329 \mathrm{em}$ setembro de 2009. A cobertura da população, contudo, ficou estável em torno de $24 \%$, nas três edições consideradas da Pnad.

No Gráfico 1 são sobrepostas representações da população total do país e da população que possui plano de saúde, segundo a forma de contratação. Para a população total se nota, entre 1998 e 2008, a concentração da população nas faixas etárias correspondentes a idade ativa, concomitantemente à diminuição da participação da população com idade até dezoito anos e ao aumento da participação de idosos na população total.

Analisando os painéis de a, b e c é possível perceber que os beneficiários de planos com contratação individual são um grupo mais envelhecido que a população total. As faixas etárias superiores a cinquenta e quatro anos representam

Tabela 2. Evolução do Índice de Envelhecimento e da Razão de Dependência

\begin{tabular}{cllllll}
\hline & & \multicolumn{4}{c}{ Anos selecionados } \\
\cline { 3 - 6 } & & 1978 & 1988 & 1998 & 2003 & 2008 \\
\hline \multirow{2}{*}{ RD } & População Total & 0,751 & 0,675 & 0,563 & 0,511 & 0,476 \\
& Beneficiários de Planos & & & 0,460 & 0,427 & 0,407 \\
& Ben. de Planos - Contratação Coletiva & & & 0,436 & 0,369 & 0,322 \\
& Ben. de Planos - Contratação Individual & & & 0,500 & 0,512 & 0,566 \\
IE $\quad$ População Total & 0,100 & 0,135 & 0,200 & 0,244 & 0,314 \\
& Beneficiários de Planos & & & 0,256 & 0,367 & 0,461 \\
& Ben. de Planos - Contratação Coletiva & & & 0,117 & 0,179 & 0,246 \\
& Ben. de Planos - Contratação Individual & & & 0,533 & 0,649 & 0,791 \\
\hline
\end{tabular}

Fonte: Elaboração própria a partir dos microdados PNAD/IBGE: 1978, 1988, 1998, 2003 e 2008. 


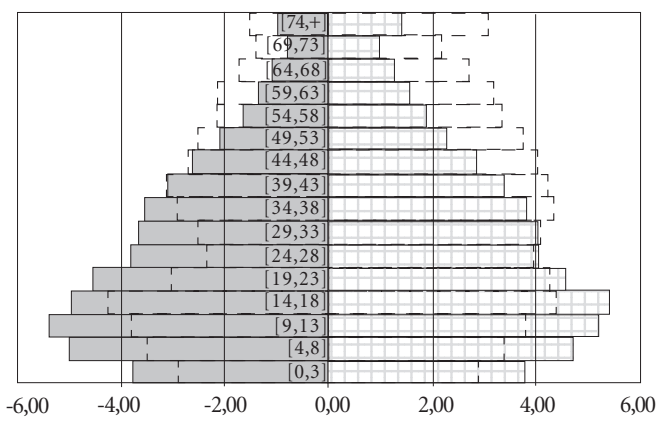

(\% da população de referência)

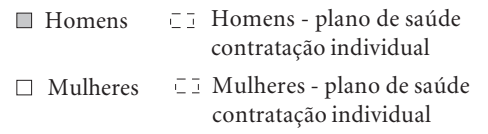

Gráfico 1a. Pirâmides Etárias - População Geral e Beneficiários de Planos de Saúde com contratação Individual - Brasil - 1998

Fonte: Elaborado a partir do microdados da PNAD-IBGE 1998.

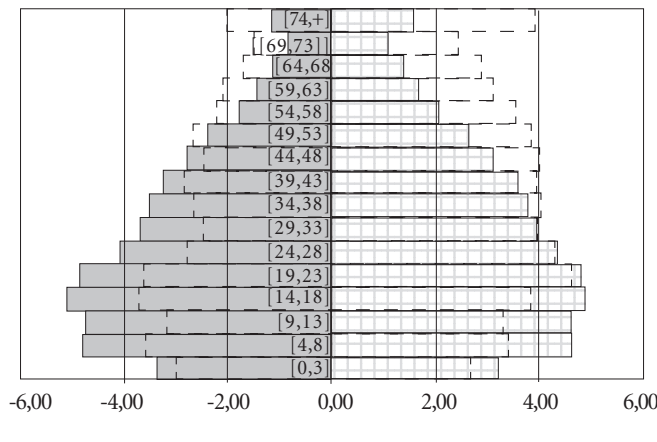

(\% da população de referência)

$$
\begin{array}{ll}
\square \text { Homens } & -\begin{array}{l}
\text { Homens - plano de saúde } \\
\text { contratação individual }
\end{array} \\
\square \text { Mulheres } & -1 \text { Mulheres - plano de saúde } \\
& \text { contratação individual }
\end{array}
$$

Gráfico 1b. Pirâmides Etárias - População Geral e Beneficiários de Planos de Saúde com contratação Individual - Brasil - 2003.

Fonte: Elaborado a partir do microdados da PNAD-IBGE 2003.

sempre uma proporção maior deste grupo de beneficiários do que da população total.

Uma situação diferente é percebida nos painéis $\mathrm{d}$, e e f, onde nota-se que o grupo de beneficiários de planos com contratação coletiva diverge da população total justamente nas faixas

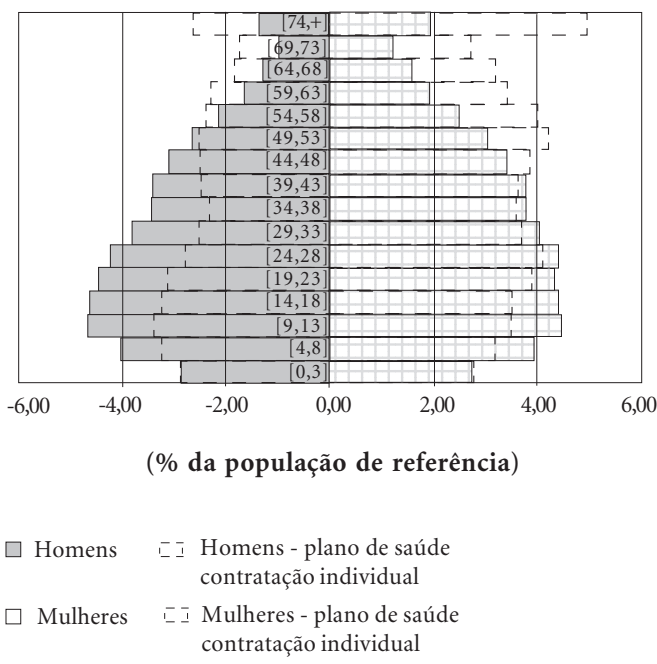

Gráfico 1c. Pirâmides Etárias - População Geral e Beneficiários de Planos de Saúde com contratação Individual - Brasil - 2008

Fonte: Elaborado a partir do microdados da PNAD-IBGE 2008.

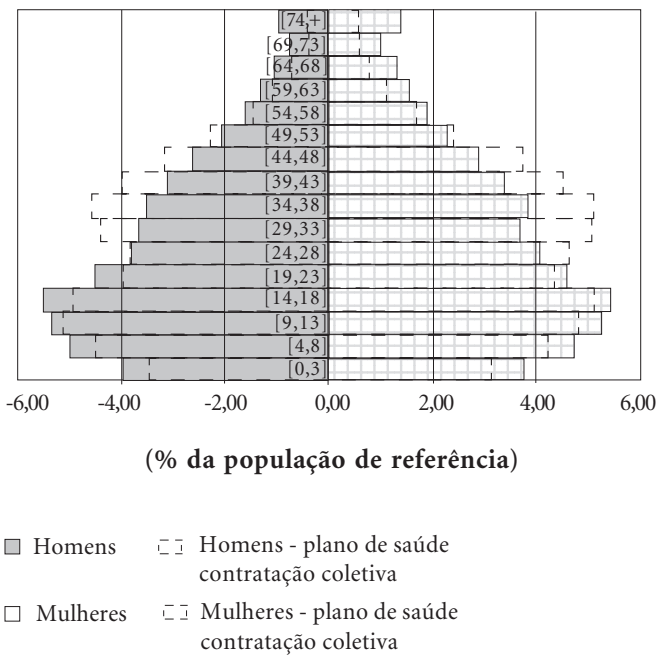

Gráfico 1d. Pirâmides Etárias - População Geral e Beneficiários de Planos de Saúde com contratação Coletiva - Brasil - 1998

Fonte: Elaborado a partir do microdados da PNAD-IBGE 1998.

etárias economicamente ativas, apresentando uma proporção inferior de jovens e idosos do que a da população total.

Os efeitos da continuidade do envelhecimento populacional sobre a saúde suplementar dependerão do quanto a população beneficiária de 


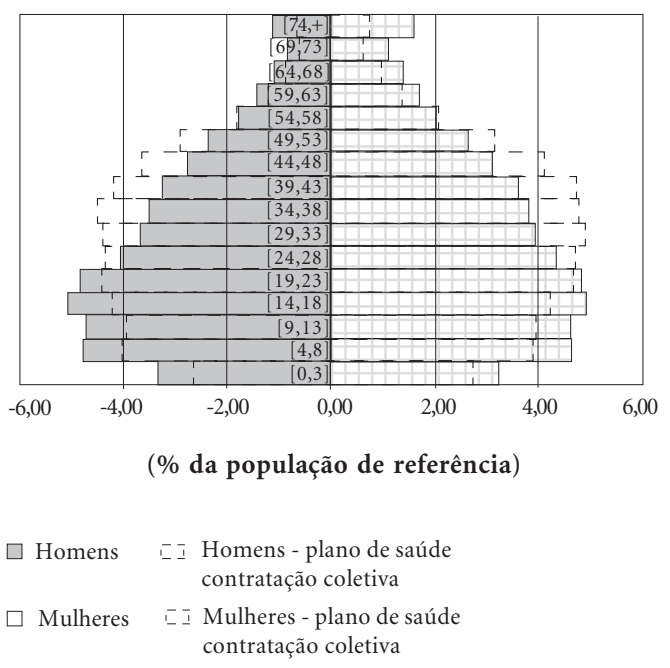

Gráfico 1e. Pirâmides Etárias - População Geral e Beneficiários de Planos de Saúde com contratação Coletiva - Brasil - 2003.

Fonte: Elaborado a partir do microdados da PNAD-IBGE 2003.

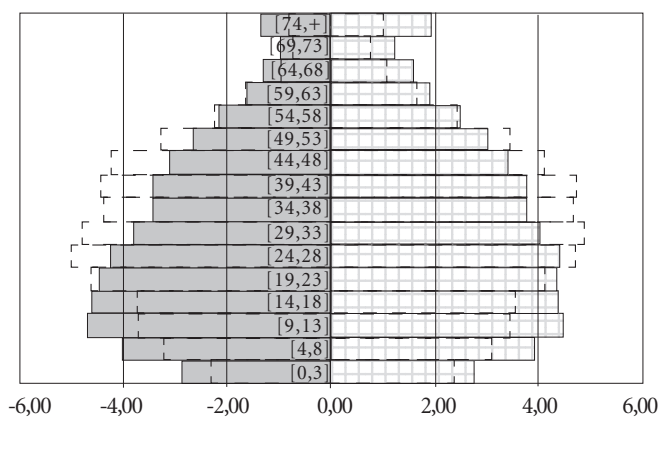

(\% da população de referência)

$$
\begin{aligned}
& \square \text { Homens } \quad \overline{-} \text { ב Homens - plano de saúde } \\
& \text { contratação coletiva } \\
& \square \text { Mulheres I- I Mulheres - plano de saúde } \\
& \text { contratação coletiva }
\end{aligned}
$$

Gráfico 1f. Pirâmides Etárias - População Geral e Beneficiários de Planos de Saúde com contratação Coletiva - Brasil - 2008.

Fonte: Elaborado a partir do microdados da PNAD-IBGE 2008.

planos de contratação individual continuará a ser mais envelhecida que a população total do país. Ou seja, dependerá de como - mais ou me- nos intensamente - essa população beneficiária acompanhará as mudanças na população geral.

Uma grande limitação da análise anterior é que ela não considerou a época de contratação dos planos, uma característica fundamental quanto à aplicação, ou não, das regras de reajuste por faixas etárias. Os planos contratados anteriormente a Lei no ${ }^{-9.656 / 98^{1}}$, e não adaptados, não são submetidos à regulação da ANS, esses planos ainda representam um contingente relevante do total. Segundo dados extraídos do ANSTabnet, em setembro de 2003, de um total de 31.340.830 de beneficiários de planos de assistência médica 15.179 .006 , ou $48 \%$, eram beneficiários de planos contratados anteriormente à Lei $\mathrm{n}^{\circ}$ 9.656/981 . Em setembro de 2008, de um total de 40.326.667 de beneficiários 10.171.113, ou 25\%, ainda representavam vínculos a planos contratados antes da Lei no ${ }^{\mathbf{9}}$ 9.656/98 ${ }^{1}$.

Não é possível identificar a época de contratação dos planos nos dados da Pnad. Para tentar contornar as deficiências da análise anterior recorre-se aos dados que a ANS disponibiliza, calculando a taxa de variação no número de beneficiários dos planos novos segundo a modalidade de contratação. Essas taxas são mostradas na Tabela 3, a seguir.

Das informações da Tabela 3 cabe destacar inicialmente que o crescimento do número de beneficiários é mais intenso nas faixas de 24 a 28 e 29 a 33 anos, independentemente da modalidade de contratação. Esse fato é esperado, já que esse intervalo corresponde à idade de ingresso no mercado de trabalho.

Adicionalmente tem-se sempre uma expansão maior nas modalidades de contratação coletiva frente à individual ao longo dos anos considerados, o que altera a participação no total de planos, mas não representa uma redução em termos absolutos nos planos de contratação individual $^{6,19}$.

Para a presente análise, cabe observar que as três últimas faixas etárias têm, frequentemente, expansão do número de beneficiários superior à observada para o total de beneficiários de planos. Contudo, enquanto o crescimento do número de beneficiários nas faixas mais altas não superarem sistematicamente os das mais jovens, não se observará o envelhecimento das carteiras das operadoras. Uma explicação possível para esse fenômeno é que o número de beneficiários idosos era menor que o número de beneficiários não-idosos anteriormente, de forma que o simples envelhecimento da população beneficiária implica um crescimento expressivo nessas faixas etárias. 
Tabela 3. Taxa de variação (\%) no número de beneficiários planos de assistência médica - Planos Novos setembro como mês de referência, segundo forma de contratação.

\begin{tabular}{|c|c|c|c|c|c|c|c|c|c|c|}
\hline & Contratação & $\begin{array}{c}2001 / \\
2000\end{array}$ & $\begin{array}{c}2002 / \\
2001\end{array}$ & $\begin{array}{c}2003 / \\
2002\end{array}$ & $\begin{array}{c}2004 / \\
2003\end{array}$ & $\begin{array}{c}2005 / \\
2004\end{array}$ & $\begin{array}{c}2006 / \\
2005\end{array}$ & $\begin{array}{l}2007 / \\
2006\end{array}$ & $\begin{array}{c}2008 / \\
2007\end{array}$ & $\begin{array}{c}2009 / \\
2008\end{array}$ \\
\hline \multirow[t]{3}{*}{00 a 18 anos } & IF & 35,96 & 13,29 & 11,06 & 17,41 & 12,13 & 6,17 & 6,11 & 2,19 & 4,46 \\
\hline & $\mathrm{CE}$ & 28,30 & 24,88 & 17,09 & 21,51 & 13,80 & 10,68 & 10,13 & 9,53 & 5,79 \\
\hline & $\mathrm{CA}$ & 28,77 & 16,29 & 23,21 & 28,08 & 11,84 & 10,44 & $-1,39$ & 11,81 & 6,97 \\
\hline \multirow[t]{3}{*}{19 a 23 anos } & IF & 31,48 & 12,35 & 8,36 & 12,87 & 8,38 & 1,63 & 4,40 & 2,47 & 4,14 \\
\hline & $\mathrm{CE}$ & 40,95 & 28,94 & 20,90 & 26,00 & 16,34 & 9,53 & 12,16 & 15,34 & 4,56 \\
\hline & $\mathrm{CA}$ & 59,08 & 10,17 & 23,00 & 19,88 & 20,14 & 8,26 & $-1,58$ & 19,93 & 4,98 \\
\hline \multirow[t]{3}{*}{24 a 28 anos } & IF & 32,85 & 16,96 & 13,54 & 14,64 & 10,45 & 5,40 & 5,72 & 1,19 & 3,81 \\
\hline & $\mathrm{CE}$ & 45,36 & 33,88 & 28,12 & 31,23 & 22,05 & 15,10 & 15,09 & 15,02 & 6,62 \\
\hline & $\mathrm{CA}$ & 46,18 & 19,72 & 28,19 & 23,84 & 28,52 & 17,71 & 3,40 & 15,96 & 6,83 \\
\hline \multirow[t]{3}{*}{29 a 33 anos } & IF & 34,33 & 17,77 & 13,61 & 14,42 & 10,01 & 6,00 & 7,38 & 5,54 & 6,94 \\
\hline & CE & 39,74 & 29,12 & 23,91 & 27,58 & 18,77 & 14,23 & 15,76 & 17,69 & 11,53 \\
\hline & $\mathrm{CA}$ & 34,30 & 19,24 & 27,14 & 22,56 & 23,39 & 18,79 & 6,42 & 18,04 & 12,55 \\
\hline \multirow[t]{3}{*}{34 a 38 anos } & IF & 34,19 & 16,20 & 11,42 & 11,19 & 9,71 & 5,03 & 5,93 & 4,41 & 5,15 \\
\hline & $\mathrm{CE}$ & 33,73 & 26,88 & 19,54 & 22,92 & 15,96 & 12,35 & 12,81 & 14,32 & 9,79 \\
\hline & $\mathrm{CA}$ & 32,70 & 20,18 & 25,42 & 18,11 & 20,07 & 15,66 & 5,24 & 14,98 & 11,11 \\
\hline \multirow[t]{3}{*}{39 a 43 anos } & IF & 36,79 & 18,63 & 15,49 & 14,54 & 11,10 & 4,26 & 4,76 & 1,41 & 2,70 \\
\hline & $\mathrm{CE}$ & 30,66 & 28,34 & 22,08 & 24,84 & 15,92 & 11,91 & 10,96 & 11,48 & 7,03 \\
\hline & $\mathrm{CA}$ & 32,19 & 24,51 & 29,08 & 22,15 & 20,88 & 15,78 & 4,59 & 11,34 & 7,29 \\
\hline \multirow[t]{3}{*}{44 a 48 anos } & IF & 35,04 & 16,33 & 14,09 & 10,26 & 8,77 & 4,00 & 7,43 & 4,24 & 4,66 \\
\hline & CE & 30,93 & 28,18 & 22,46 & 23,34 & 15,13 & 13,05 & 12,65 & 14,29 & 9,57 \\
\hline & $\mathrm{CA}$ & 29,38 & 24,11 & 27,22 & 21,40 & 19,93 & 17,49 & 7,58 & 14,59 & 9,92 \\
\hline \multirow[t]{3}{*}{49 a 53 anos } & IF & 38,54 & 18,97 & 19,37 & 15,01 & 13,04 & 4,72 & 7,29 & 2,15 & 3,56 \\
\hline & $\mathrm{CE}$ & 33,42 & 28,15 & 26,27 & 25,22 & 17,59 & 15,22 & 13,51 & 14,18 & 9,95 \\
\hline & $\mathrm{CA}$ & 29,96 & 22,87 & 29,52 & 22,25 & 19,65 & 18,61 & 8,56 & 13,41 & 8,34 \\
\hline \multirow[t]{3}{*}{54 a 58 anos } & IF & 35,35 & 17,97 & 19,48 & 17,37 & 15,42 & 7,83 & 10,76 & 6,60 & 6,45 \\
\hline & $\mathrm{CE}$ & 31,89 & 27,56 & 26,48 & 28,15 & 17,96 & 16,71 & 13,11 & 16,18 & 11,88 \\
\hline & $\mathrm{CA}$ & 30,30 & 24,03 & 31,07 & 25,69 & 16,96 & 19,57 & 11,24 & 14,99 & 8,82 \\
\hline \multirow{3}{*}{$\begin{array}{l}59 \text { anos ou } \\
\text { mais }\end{array}$} & IF & 29,95 & 11,90 & 21,55 & 16,06 & 15,20 & 9,71 & 11,98 & 7,64 & 11,24 \\
\hline & $\mathrm{CE}$ & 18,46 & 14,70 & 23,71 & 21,89 & 14,09 & 14,10 & 10,52 & 13,26 & 11,08 \\
\hline & CA & 33,65 & 22,62 & 27,86 & 25,15 & 12,39 & 18,46 & 14,18 & 11,46 & 11,47 \\
\hline \multirow[t]{3}{*}{ Inconsistente } & IF & 20,34 & 7,93 & 11,37 & $-7,08$ & $-36,20$ & $-34,80$ & $-27,74$ & $-31,78$ & $-15,53$ \\
\hline & $\mathrm{CE}$ & 2,48 & 4,62 & 5,72 & 6,62 & $-0,48$ & $-5,18$ & $-9,05$ & $-3,90$ & $-4,09$ \\
\hline & $\mathrm{CA}$ & 3,87 & 2,12 & 3,02 & $-2,59$ & $-24,07$ & $-14,46$ & $-12,15$ & $-14,66$ & $-11,48$ \\
\hline \multirow[t]{3}{*}{ Total } & IF & 34,43 & 15,03 & 13,69 & 15,09 & 11,55 & 5,79 & 7,01 & 3,51 & 5,47 \\
\hline & $\mathrm{CE}$ & 32,23 & 26,61 & 21,49 & 24,54 & 16,29 & 12,55 & 12,30 & 13,34 & 8,00 \\
\hline & $\mathrm{CA}$ & 34,59 & 19,19 & 26,21 & 23,77 & 17,90 & 15,02 & 4,68 & 14,15 & 8,72 \\
\hline
\end{tabular}

Legenda: $\mathrm{IF}=$ Individual ou Familiar, $\mathrm{CE}=$ Coletivo Empresarial e $\mathrm{CA}=$ Coletivo por adesão.

Fonte: Elaboração própria a partir de dados de ANS Tabnet

Os resultados até aqui mostram que embora a população brasileira esteja envelhecendo, em especial a parte da população que é beneficiária de planos de saúde, não se pode perceber uma mudança no sentido da regulação induzir que os consumidores mais jovens abandonem a saúde suplementar. Contudo, pode-se notar uma tendência à sobrerrepresentação de idosos em planos de contratação individual ou familiar, tanto nos dados da Pnad quanto nos da ANS. Em especial, para os planos assinados após a Lei nº 9.656/981, cujo número de beneficiários na última faixa etária considerada cresce acima das demais faixas.

Uma avaliação adicional ainda pode ser feita considerando as implicações, em termos de despesas, do envelhecimento populacional. Para tanto a transição epidemiológica ${ }^{20}$ também é relevante, pois representa uma mudança na estrutura de despesas relacionadas aos tratamentos de saúde. Vários estudos apontam que os cuidados 
à saúde da população idosa demandam mais recursos que os cuidados da população mais jovem $^{21-25}$, seja pelos idosos utilizarem com uma frequência maior os serviços, seja pela incidência de doenças crônico-degenerativas, de maior predominância nos idosos, demandar cuidados mais intensivos e dispendiosos.

No caso do Brasil, trabalhos que identificam essa mudança nos padrões de morbidade e mortalidade ${ }^{26,27}$ destacam que se enfrenta no país uma transição incompleta, que corresponderia a uma mudança no padrão de morbimortalidade, com aumento da importância de doenças crônicas não-transmissíveis, mas ainda com a persistência de doenças infecciosas.

Utilizando os dados da Pnad pode-se realizar o cálculo da prevalência das morbidades e avaliar sua evolução entre 1998 e 2008, contudo esses valores devem ser interpretados considerando as ressalvas já feitas às limitações dos dados. Selecionando apenas três morbidades, nota-se que entre beneficiários de planos de saúde a prevalência de: (i) câncer passou de 0,28\% em 1998, para $0,64 \%$ em 2003 e para $0,86 \%$ em 2008; (ii) diabetes passou de 2,46\% em 1998, para 3,23 em 2003, e para 4,29\% em 2008; e (iii) hipertensão passou de 11,37\% em 1998, para 13,66\% em 2003, e para $15,79 \%$ em 2008. Comportamento similar a esse, em nível e trajetória, é observado para a população não vinculada a planos de saúde.

Parte da elevação das prevalências associadas a essas doenças crônicas poderia ser atribuída à mudança na forma como o entrevistado era questionado sobre a morbidade entre 1998 e 2003. Contudo, essa explicação não se aplica entre 2003 e 2008. O crescimento das prevalências é substancial, correspondendo a um forte indicativo da transição epidemiológica.

A tendência observada do aumento da importância das doenças crônico-degenerativas na população brasileira tem impacto direto sobre a demanda por cuidados à saúde e sobre as despesas envolvidas, tanto para o atendimento via saúde suplementar quanto para o atendimento no SUS.

\section{Considerações Finais}

É comum que os beneficiários de planos de saúde percebam o reajuste por faixas etárias como um mecanismo prejudicial aos idosos e não o contrário. Por outro lado, também é comum que as operadoras de planos fiquem insatisfeitas com a regulação que a proíbe de cobrar mais dos beneficiários que estão associados a maiores custos.

A variação das mensalidades (contraprestações) dos planos de saúde segundo as faixas etárias dos beneficiários impõe um elemento de solidariedade ao financiamento da saúde suplementar. No gradiente possível entre eficiência plena (discriminação total de risco) e equidade plena (community rating puro) as faixas representam uma escolha intermediária que permite o acesso a cuidados à saúde para várias pessoas, que em outro esquema de financiamento estariam excluídas por serem incapazes de pagarem pelo serviço, sem expulsar do sistema os beneficiários mais saudáveis.

A mudança da estrutura etária da população brasileira, passando a uma situação com maior predominância de idosos nos próximos anos, apresenta um desafio para a manutenção dessa regra. O envelhecimento populacional significa que o grupo com maiores despesas no sistema aumentará sua participação, exigindo um subsídio maior dos mais jovens.

Os dados analisados mostram que há um envelhecimento populacional mais intenso entre os beneficiários de planos de saúde e também que essa mudança demográfica não implicou uma mudança no sentido dos jovens abandonarem a saúde suplementar. Uma explicação possível para não se observar esse resultado, ao menos para os planos de contratação coletiva/empresarial, é que a decisão quanto à contratação ou não do plano é tomada pelo empregador e não pelo empregado/beneficiário, para o qual o plano de saúde corresponde a um "fringe benefit" do emprego que não pode ser substituído por um salário monetário maior.

Há espaço para o elemento de equidade no sistema de seguro-saúde, contudo ele é limitado. Conforme exposto por Arrow ${ }^{28}$ o consumidor que compra um plano de saúde, devido a sua aversão ao risco, também o comprará por um preço superior ao atuarialmente justo, desde que esse preço não seja muito superior.

Com a intensificação do processo de envelhecimento as regras serão tensionadas demandando alguma revisão, especificamente no limite imposto para última faixa etária pela Lei no 10.741/03. É fundamental que se traga para as atribuições da ANS a capacidade de rever essas regras, ainda que sob supervisão do Legislativo, pois é a Agência que zela pela saúde suplementar, e é ela que tem elementos para avaliar o trade-off entre equidade e eficiência nesse mercado. 


\section{Referências}

1. Brasil. Lei no 9.656 de 3 de junho de 1998. Dispõe sobre os planos e seguros privados de assistência à saúde. Diário Oficial da União 1998; 3 jun.

2. Brasil. Lei no 10.741 de 1 de outubro de 2003. Dispõe sobre o Estatuto do Idoso e dá outras providências. Diário Oficial da União 2003; 1 out.

3. Cutler DM, Zeckhauser RJ. The Anatomy of Health Insurance. In: Culyer AJ, Newhaouse JP, organizadores. Handbook of Health Economics. Amsterdam: North-Holland; 2000. p. 563-643.

4. Rothschild M, Stiglitz J. Equilibrium in Competitive Insurance Markets: An Essay on the Economics of Imperfect Information. Q J Econ 1976; (90)4: 629-649.

5. Cechin J, organizador A história e os desafios da saúde suplementar - 10 anos de regulação. São Paulo: Saraiva / Letras \& Lucros; 2008.

6. Santos FP, Malta DC, Mehry EE. A regulação na saúde suplementar: uma análise dos principais resultados alcançados. Cien Saude Colet 2008; (13)5: 1463-1475.

7. Montone J. Planos de Saúde: passado e futuro. Rio de Janeiro: MedBook; 2009.

8. Smith PC, Witter SN. Risk Pooling in Health Care Financing: The Implications for Health System Performance. Washington: World Bank, 2004. (Health, Nutrition and Population Discussion Paper).

9. Buchmueller T, Dinardo J. Did Community Rating Induce an Adverse Selection Death Spiral? Evidence from New York, Pennsylvania, and Connecticut. Am Econ Rev 2002; (92)1:280-294.

10. Slivak JS. Documento Técnico Contendo o Mapeamento das Cláusulas de Reajuste dos Produtos com Registro Definitivo na ANS. Rio de Janeiro: ANSDIPRO; 2007. (Pnud Projeto BRA/05/027).

11. Farias LO, Melamed C. Segmentação de mercados da assistência à saúde no Brasil. Cien Saude Colet 2003; 8(2):585-598.

12. Bahia L, Luiz RR, Salm C, Costa AJL, Kale PL, Cavalcanti MLT. O mercado de planos e seguros de saúde no Brasil: uma abordagem exploratória sobre a estratificação das demandas segundo a PNAD 2003. Cien Saude Colet 2006; 11(4):951-965.

13. Barros MBA, César CLG, Carandina L, Torre GD. Desigualdades sociais na prevalência de doenças crônicas no Brasil, PNAD-2003. Cien Saude Colet 2006; 11(4):911-926.

14. Jardim R, Barreto SM, Gonçalves LG. Confiabilidade do informante secundário em inquéritos de saúde. Rev Bras Estud Popul 2009; 26(1):141-144.

15. Carvalho JAM, Garcia RA. O envelhecimento da população brasileira: um enfoque demográfico. Cad Saude Publica 2003; (19)3:725-733.

16. Alves JED, Vasconcelos DS, Carvalho AA. Estrutura etária, bônus demográfico e população economicamente ativa no Brasil: cenário de longo prazo e suas implicações para o mercado de trabalho. Brasília: Cepal-Escritório no Brasil/Ipea; 2010. (Texto para Discussão Cepal-Ipea, n. 10).
17. Bloom DE, Canning D, Sevilla J. The Demographic Dividend: a new perspective on the economic consequences of population change. Santa Monica: RAND; 2003.

18. Mason A. Demographic Transition and Demographic Dividends in Developed and Developing Countries. In: Proceedings of the United Nations Expert Group Meeting on Social and Economic Implications of Changing Population Age Structures; 2005; Ciudad del Mexico.

19. Agência Nacional de Saúde Suplementar (ANS). Caderno de Informação da Saúde Suplementar : beneficiários, operadoras e planos da saúde. (Dezembro de 2009). Rio de Janeiro: ANS; 2009.

20. Omran AR. The Epidemiologic Transition: A Theory of the Epidemiology of Population Change. Milbank Mem Fund Quart 1971; (49)4:509-538.

21. Brito RJA, Andrade MV, Perpétuo IH. O. Demanda por Serviços de Saúde. In: Silveira FG, Servo LMS, Menezes T, Piola SF, organizadores. Gasto e Consumo das Famílias Brasileiras Contemporâneas. Brasília: IPEA; 2007. p. 345-374.

22. Lubitz J, Beebe J, Baker C. Longevity and Medicare Expenditures. N Engl J Med 1995; (332)15:999-1003.

23. Miller T. Increasing Longevity and Medicare Expenditures. Demography 2001; (38)2:215-226.

24. Nunes A. Os custos do tratamento de saúde dos idosos no Brasil. In: Camarano AA, organizadora. Muito além dos 60 - Os novos idosos brasileiros. Rio de Janeiro: IPEA; 1999. p. 345-366.

25. Nunes A. O Envelhecimento Populacional e as Despesas do Sistema Único de Saúde. In: Camarano AA, organizadora. Os novos idosos brasileiros Muito além dos 60?. Rio de Janeiro: IPEA; 2004. p. 427-450.

26. Bobadilla JL, Possas, CA. How the Epidemiological Transition Affects Health Policy Issues in Three Latin American Countries. Washington: World Bank; 1992. (Working Paper 987).

27. Prata PR. A Transição Epidemiológica no Brasil. Cad Saude Publica 1992; (8)2:168-175.

28. Arrow KJ. Uncertainty and the Welfare Economics of Medical Care. Am Econ Rev 1963; (53)5:941-973.

Apresentado em 23/09/2010

Aprovado em 22/05/2011

Versão Final apresentada em 20/06/2011 